\title{
Work-related Stress and Urinary Catecholamines among Laboratory Technicians
}

\author{
Ali GHadDar ${ }^{1,2}$, Kayan Hajj OMAR ${ }^{3}$, Maha DoKmaK ${ }^{3}$, Nadine Abou Kansour ${ }^{3}$, \\ Zeina JBARA ${ }^{3}$, Sandy LAHAM ${ }^{1,2}$ and Ali SAMAHA ${ }^{1-4}$
}

${ }^{1}$ Observatory of Public Policies \& Health, Center Bonmarche, Lebanon, ${ }^{2}$ Lebanese International University, Department of Biomedical Sciences, Lebanon, ${ }^{3}$ Lebanese University, Faculty of Public Health IV, Lebanon and ${ }^{4}$ Beirut Arab University, Faculty of Medicine, Lebanon

\begin{abstract}
Work-related Stress and Urinary Catecholamines among Laboratory Technicians: Ali GHADDAR, et al. Observatory of Public Policies and Health, Center Bonmarche, Lebanon-Objectives: Workrelated psychosocial hazards are associated with adverse health outcomes among workers. The association between psychosocial hazards and physiological health outcomes among laboratory technicians has not been studied previously. The objective of this study was to measure the association between work-related psychosocial hazards and the level of urinary catecholamines of laboratory technicians. Methods: The Copenhagen Psychosocial Questionnaire was distributed to a sample of 125 workers in one community in Lebanon (response rate $73 \%$ ) to measure psychosocial hazards. High-pressure liquid chromatography (HPLC) was used to measure adrenaline, noradrenaline and dopamine in 24-hour urine samples. Results: Sensorial demands, insecurity at work, influence at work and low possibilities of development were particularly high among the workers. Linear regression analysis showed that sensorial and quantitative demands and low possibilities for development increased the levels of urinary catecholamines. Discussion: the results suggest important policy implications for laboratory administrations regarding improvement of the exposure of workers to sensorial and quantitative demands and low possibilities for development as a way to improve worker health.
\end{abstract}

(J Occup Health 2013; 55: 398-404)

Key words: Catecholamine, Laboratory technician, Psychosocial, Stress

All the authors involved in this project declare that

Received Mar 5, 2013; Accepted May 24, 2013

Published online in J-STAGE Jul 26, 2013

Correspondence to: Ali Samaha, Coordinator of Biomedical Sciences, Lebanese International University, Mseitbeh, 08834 Beirut, Lebanon, (email: ali.samaha@liu.edu.lb) there is no conflict of interest and meet the relevant authorial criteria. The article is original and has not been sent to any other journal for publication. Authors confirm that written patient consent is available for participants.

Increasing concern about the role of the psychosocial environment at work in shaping health among workers has been noted during the last few decades ${ }^{1,2)}$. The demand- control-support theory ${ }^{3)}$ and the effortreward imbalance theory ${ }^{4}$ have been the most commonly used theoretical frameworks that explained the effects of psychosocial work environment on physiological and psychological health reactions among workers.

This issue has been examined in a wide variety of occupations $^{5)}$; however, the relationship between workrelated psychosocial stress and health among the laboratory technicians has still not been well investigated. As a matter of fact, laboratory technicians are subject to a harsh psychosocial work environment. Their daily tasks imply high exposures to psychological demands (sensorial and cognitive), as they are required to handle precisely potentially hazardous infectious biological specimens, which poses a significantly increased risk to their health ${ }^{6)}$. Moreover, the laboratory technicians' tasks involve great responsibility, as they deal with the clinical diagnosis of patients ${ }^{7}$. Besides, like any large bureaucratic institution, laboratories tend to be monotonous and impersonal, which increases the staff's alienation and feeling of lack of significance or participation.

Little has been published in the scientific literature about the health of laboratory technicians and their exposures to occupational stress. One study that described the mortality rates, causes and distribution among laboratory technicians in Britain showed that they have higher mortality rates than other health- 
care providers $^{8}$. In fact, most published studies about laboratory technicians have focused on means of improving their occupational safety and their protection against work accidents ${ }^{6}$. Other studies have examined organizational behavioral concerns among laboratory technicians such as job satisfaction and absenteeism $^{9}$. Besides, some studies have described infectious diseases as the most common problems caused by laboratory work and showed that the highest incidence rates of occupational Hepatitis C occurred in laboratory technicians in comparison with other hospital workers ${ }^{10)}$. Nevertheless, a systematic review of the theme revealed that only few published studies about work-related stress and health among laboratory technicians adopted self-perceived measures of health (depression) and used objective indicators of health status $^{11,12)}$. Although self-perceived health indicators have been commonly used in the scientific literature as proxy measures of work-related stress outcomes ${ }^{1,2)}$, it is well known that objective physiological measures for stress outcomes such as stress urinary hormones levels ${ }^{13,14)}$ are still preferred for their better reliability $^{7}$. Recent epidemiological studies revealed that urinary adrenaline, noradrenaline and dopamine of workers were higher in workers with long shifts ${ }^{15}$ and after exposure to heat ${ }^{16)}$. These studies were based on small samples composed of students and of workers from a manufacturing company and did not explore the psychosocial work environment and work-related stress.

It is thus considered of high importance to add objective evidence and knowledge based on clinical health indicators to already known theory about suggested associations between health and work-related psychosocial hazards in workers from diverse occupations. The objective of this study was to measure the association between work-related psychosocial hazards and the levels of urinary catecholamines of laboratory technicians. It was hypothesized that high exposures to work-related psychosocial hazards is associated with a biological response categorized by an increase in the urinary levels of adrenaline, noradrenaline and dopamine of the workers.

\section{Materials and Methods}

The study sample constituted workers from 12 medical laboratories in one community in Lebanon (125 workers or around $6 \%$ of laboratory technicians in the country). In Lebanon, laboratories are divided into private laboratories and public hospital laboratories, in which the design and organisation of work varies. For this study, private and small specialized laboratories were excluded, as they have different structures and work organizations compared with usual hospital laboratories. A pilot study was conducted among 7 laboratory technicians to adapt the questionnaire to the workplace particularities and dynamics.

A multistage sampling procedure was used to select a sample of 12 laboratories from the total of 20 laboratories in a province in Lebanon in the first stage and a random sample of 11 technicians from each laboratory in the second stage (total sample population=132). Seven workers had less than one year at work and were thus excluded, as they had little exposure to psychosocial hazards at work. From the 125 workers, 34 workers refused to answer the survey, yielding a response rate of $73 \%$.

The study proposal was approved by the university's ethics review committee (approval number: UL-FSPIV-SL-10-3). Oral informed consent was obtained from each participant during the period of urine sample collection. A self-administered questionnaire consisting of 95 questions was used to measure psychosocial hazards and sociodemographic and work-related variables. Factors related to psychosocial work environment were measured using the Copenhagen Psychosocial Questionnaire (COPSOQ), a comprehensive and validated tool for work and health ${ }^{17)}$. The reliability, validity, and applicability of the original version have been discussed (Cronbach's alphas for items ranging between 0.69 and 0.91$)^{17)}$. Based on the results of the pilot study, seventeen of the original twenty-one risks were selected: five scales on demands (quantitative demands, cognitive demands, sensorial demands, emotional demands and demands for hiding emotions), four scales on control and active developmental work (control over time, influence on work, possibilities for development and meaning of work), two scales on social support (social support from colleagues and superiors), one scale on job insecurity and one scale on self-esteem. Responses used Likert-type scales that were formed by summing the points of the individual questions, giving equal weight to each question, dividing the sum by the maximum score possibly obtained and finally multiplying by 100. Scores ranged from 0 (minimum exposure) to 100 (maximum exposure). Results for each of the work-related psychosocial hazards were expressed in three categories using as the calculated tertiles of the sample population as cut-off values. For example, after calculating the tertile cut points for the quantitative demand (31.25 and 43.75), laboratory technicians were classified into one of three categories according to their scores. Those with scores lower than 31.25 were classified in the "favorable interval", those with scores between 31.25 and 43.75 were classified in the intermediate interval, and those with scores higher than 43.75 were classified in the risk interval.

The most central background variables, such as sex, age and education (elementary, secondary, univer- 
sity and post-graduate), were chosen as moderating individual factors. Other job characteristics, such as years at work (defined as the total number of years at work in laboratories) and present occupational group, were included, since their impact on stress among workers was discussed in previous studies ${ }^{2}$. Group intervals for age (years) were defined as 26-40, $40-45,45-50$ and $>50$, and for years at work (years), the group intervals were defined as 1-12, 12-20 and $>20$, containing similar percentages of participants in each group. Participants were asked about their smoking habits (never smoked vs. previous or current smoker) and the number of cigarettes smoked per day. Before starting collection of data, information on the study's context and aim was conveyed to the workers. Participation was voluntary and confidential. The method used for data collection for the independent variables (psychosocial risks) was self-administered anonymous questionnaires. Questionnaires were distributed in each laboratory during May 2011 in cooperation with the representatives of the workers during 4 consecutive days. Workers either completed the questionnaire immediately or completed them at home. They were asked to leave the answered questionnaire in a sealed box present in the laboratory reception area.

In our study, catecholamines in the urine (noradrenaline, adrenaline and dopamine) were measured as a biological response to stress. Urine samples were collected using the 24-hour urine collection method, which is recommended for the measurement of catecholamines in urine ${ }^{18)}$. From a physiological standpoint, stress hormones fluctuate significantly during the day. These fluctuations make the measurement of adrenal activity imprecise with a single observation during the day and night, which makes it preferable to collect 24-hour urine for assay. Urine sample were collected during 4 consecutive days in each laboratory in opaque containers, as catecholamines are sensitive to light, and were kept at $4{ }^{\circ} \mathrm{C}$ and sent to the Immunological Center of Catalonia, Spain, for analysis by high pressure liquid chromatography (HPLC). Cronbach's alphas were calculated to check the internal consistency of the work-related psychosocial hazards (with an alpha of 0.70 or higher indicating satisfactory internal consistency).

First, the distribution of laboratory technicians in risk intervals (risk vs. no risk) of psychosocial hazards was compared between males and females (Chi-square test). Then, the correlation between the individual psychosocial hazards at work with urine catecholamine scores (continuous dependent variables) was explored using linear regression analysis (SPSS version 15). The model was constructed in three stages. The first stage consisted of selecting only the most relevant work-related psychosocial hazards (in one domain) and the most significant control variables such as sex, age, professional experience and shift work (in another domain) for further inclusion in the final model. For this aim, linear regression was applied to these variables, and only those that had $p<0.05$ were retained. The second stage consisted of selecting the variables in each domain for inclusion in the final model. For this purpose, linear regression was applied separately to each domain, and only the variables with $p<0.05$ were retained. The last stage was that of constructing the final model on the basis of the variables preselected during the first two stages. Psychosocial hazards followed by the control variables (only with $p<0.05$ ) were entered into the model in the following order: psychosocial hazards, gender, educational level and shift work.

\section{Results}

The personal and work-related characteristics of the participants $(n=91)$ are presented in Table 1.

Internal consistencies of the dimensions in the studied sample (as measured by the Cronbach's alpha coefficient) were between 0.55 and 0.87 for the dimensions of the psychosocial hazards at work. Figure 1 presents the frequency and percentage of workers in each group (favorable, intermediate and risk) according to their exposure to each of the studied psychosocial hazards. Respondents reported overall high to moderate levels of exposures to the studied work-related psychosocial hazards when compared with the standard population (Fig. 1), with the exception of cognitive demands $(5.5 \%)$, emotional demands $(0 \%)$, demands for hiding emotions $(0 \%)$, quality of leadership (3.3\%), social support $(8.8 \%)$ and sense of

Table 1. Sociodemographic characteristics of the sample

\begin{tabular}{lc}
\hline & $\mathrm{n}(\%)$ \\
\hline Gender & \\
Male & $29(32.0)$ \\
Female & $62(68.0)$ \\
Age & \\
$20-25$ & $25(27.5)$ \\
$25-30$ & $31(34.1)$ \\
$30-40$ & $25(27.5)$ \\
$>40$ & $10(11.0)$ \\
Education level & \\
Bachelor of science & $53(58.2)$ \\
Bachelor of technology & $37(40.7)$ \\
Marital status & \\
Single & $50(54.9)$ \\
Married & $41(45.1)$ \\
\hline
\end{tabular}


a High $\square$ Intermediate $\square$ Favorable

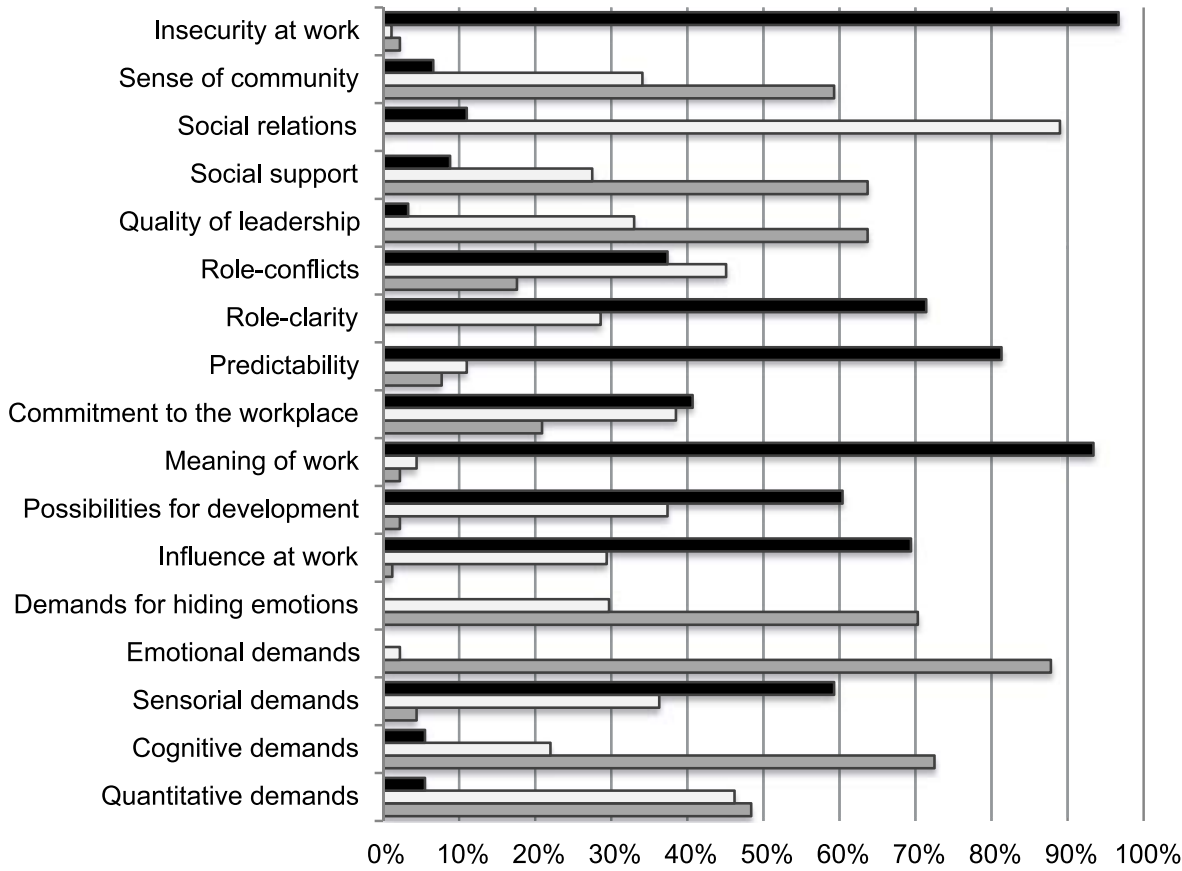

Fig. 1. Distribution of laboratory technicians in risk intervals (favorable, intermediate and risk) according to their exposure to work-related psychosocial hazards.

community $(6.6 \%)$.

The mean and standard deviation of the catecholamine levels in the urine were 5.66 and 0.32 for adrenaline, 65.39 and 3.41 for noradrenaline and 565.65 and 61.17 for dopamine, respectively. The reference values in the population are estimated to be less than $10 \mu \mathrm{g}$ for adrenaline, less than $100 \mu \mathrm{g}$ for noradrenaline and less than $400 \mu \mathrm{g}$ for dopamine for 24-h urine samples collected from healthy adults ${ }^{19}$.

In single regression analysis (Table 2), adrenaline levels in the urine had positive significant associations with quantitative demands, sensorial demands and influence at work and negative significant associations with demands for hiding emotions, possibilities for development and role-conflicts. On the other hand, noradrenaline levels in the urine were positively associated with quantitative and sensorial demands and influence at work, while they had negative significant associations with cognitive demands, demands for hiding emotions, emotional demands, possibilities for development, role conflict and social support. Moreover, dopamine levels had significant positive associations with quantitative and sensorial demands and negative significant associations with possibilities for development.
After adjusting for the rest of the psychosocial hazards and the main control variables (gender, age and shift work), multiple regression analysis (Table 2) showed that quantitative and sensorial demands increased and possibilities for development decreased the levels of all three studied catecholamines (statistically significant associations). In addition, it is worth mentioning that although workers perceived that they had low influence at work (69.4\% at risk), the association between low influence at work and adrenaline, noradrenaline and dopamine levels in the urine was statistically nonsignificant after adjusting for the rest of the control variables.

It is worth mentioning that although females had higher levels of adrenaline and dopamine in the urine, the association between gender and catecholamine levels in the urine was not statistically significant. Similarly, despite the fact that shift work increased the catecholamine levels in the urine (beta=1.12 and 0.74 for noradrenaline and dopamine respectively), the association was not statistically significant.

\section{Discussion}

The present study suggested that laboratory technicians have high exposures to work-related psychoso- 
Table 2. Results of the linear regression model with catecholamine variables as dependent variables and psychosocial risks, gender, age and shift work as independent variables

\begin{tabular}{|c|c|c|c|c|c|c|c|c|c|}
\hline & \multicolumn{3}{|c|}{ Adrenaline } & \multicolumn{3}{|c|}{ Noradrenaline } & \multicolumn{3}{|c|}{ Dopamine } \\
\hline & $\begin{array}{l}\text { Beta } \\
\text { (crude) }\end{array}$ & $\begin{array}{c}\text { Beta } \\
\text { (adjusted) }\end{array}$ & $95 \% \mathrm{CI}$ & $\begin{array}{l}\text { Beta } \\
\text { (crude) }\end{array}$ & $\begin{array}{c}\text { Beta } \\
\text { (adjusted) }\end{array}$ & $95 \% \mathrm{CI}$ & $\begin{array}{l}\text { Beta } \\
\text { (crude) }\end{array}$ & $\begin{array}{c}\text { Beta } \\
\text { (adjusted) }\end{array}$ & $95 \% \mathrm{CI}$ \\
\hline Quantitative demands & $0.88^{*}$ & $0.71^{*}$ & $0.32 ; 0.66$ & $2.98 * *$ & $2.73 *$ & $2.21 ; 3.89$ & $65.38^{* *}$ & $31.87 *$ & $24.33 ; 51.14$ \\
\hline Sensorial demands & $0.07 *$ & $0.22 * *$ & $0.49 ; 3.23$ & $1.57^{*}$ & $1.32 *$ & $0.19 ; 1.24$ & $4.93 * *$ & $3.76 * *$ & $345.21 ; 458.23$ \\
\hline Cognitive demands & -0.24 & -0.06 & $-0.19 ; 0.01$ & $-0.66^{*}$ & $-0.31^{*}$ & $0.82 ; 1.63$ & 0.67 & 0.48 & $-175.69 ; 382.68$ \\
\hline $\begin{array}{l}\text { Demands for hiding } \\
\text { emotions }\end{array}$ & $-0.10 * * *$ & $-0.04 * *$ & $-0.06 ;-0.00$ & $-0.49 * *$ & $-0.39 * *$ & $-0.41 ;-0.66$ & 0.77 & 0.91 & $-312.71 ; 321.54$ \\
\hline Emotional demands & -0.21 & -0.19 & $-0.24 ; 0.32$ & $-3.12 * *$ & -3.41 & $-5.33 ; 2.21$ & 0.86 & 0.34 & $-434.26 ; 581.06$ \\
\hline Influence at work & $0.11^{*}$ & 0.31 & $0.11 ; 0.61$ & $0.44 *$ & 0.79 & $0.54 ; 1.99$ & 1.65 & 1.33 & $-129.12 ; 501.28$ \\
\hline $\begin{array}{r}\text { Possibilities for } \\
\text { development }\end{array}$ & $-0.08^{*}$ & $-0.37 * *$ & $-0.31 ;-0.09$ & $-2.84 *$ & $-2.61 *$ & $-3.28 ;-2.65$ & $-1.07^{*}$ & $-1.46^{* *}$ & $-12.18 ;-1.76$ \\
\hline Role-conflicts & $-0.09 *$ & $-0.32 * *$ & $-0.32 ;-0.03$ & $-2.21 * * *$ & $-1.98^{*}$ & $-2.38 ;-1.32$ & -0.45 & -0.31 & $-53.19 ; 112.36$ \\
\hline Social support & -0.08 & -0.03 & $-0.16 ; 0.19$ & $-1.84 * *$ & $-1.12^{*}$ & $-2.21 ;-1.12$ & 0.99 & 0.67 & $-401.48 ; 538.14$ \\
\hline Insecurity at work & 0.04 & -0.05 & $-0.38 ; 0.12$ & -0.42 & -0.22 & $-0.69 ; 1.59$ & 0.81 & 0.21 & $-372.82 ; 310.91$ \\
\hline Gender & 0.06 & 0.11 & $-0.51 ; 0.76$ & -127 & -0.56 & $-3.42 ; 2.21$ & 0.65 & 0.53 & $-1267 ; 2746.12$ \\
\hline Age & 0.27 & 0.23 & $-0.74 ; 1.22$ & 0.53 & 0.31 & $-5.35 ; 17.66$ & 0.40 & 0.29 & $-103.51 ; 248.66$ \\
\hline Study & 0.41 & 0.37 & $-0.26 ; 1.31$ & 2.48 & 1.33 & $-0.56 ; 4.12$ & 1.922 & 1.01 & $-1262 ; 2303.15$ \\
\hline Shift & 0.18 & -0.23 & $-1.13 ; 0.78$ & 1.97 & 1.12 & $-5.22 ; 2.32$ & 0.74 & 0.36 & $-0.83 ; 70.31$ \\
\hline
\end{tabular}

Beta (crude): crude non-standardized single regression coefficient. Beta (adjusted): adjusted non-standardized multiple regression coefficient. $* p<0.05 ; * * p<0.01 ; * * * p<0.001$.

cial hazards. The findings also highlighted the role of certain elements of the psychosocial environment in predicting ill health in a newly-studied high-risk work environment. The results were consistent with findings of previous studies in another work environment that highlighted a positive significant association between shift work and increased urinary levels of adrenaline, noradrenaline and dopamine ${ }^{15}$. They were also consistent with results of previous studies in other countries ${ }^{11)}$ that identified the same work-related psychosocial hazards (low control of work, quantitative demands and low decision authority) among the major predictors of stress and depression among laboratory technicians. Likewise, the results of this study agreed with previous reviews that concluded that "urinary catecholamines appear to reflect the effects of shift work and monotonous work"20).

The results, however, showed interesting and surprising findings, for example, that there were no statistically significant correlations between emotional demands and levels of catecholamines in the urine. It is also worth noting that cognitive demands were associated with decreased levels of noradrenaline in the urine (beta $=-0.31, p=0.04$ ), which suggests that cognition is associated with better health.

The results have important policy implications for laboratory administrations, especially in terms of reducing the major psychosocial risks at work that were associated with poor workers' health. For example, it could be highly beneficial to reduce the exposures of workers to low possibilities for development: $60 \%$ of workers perceived that laboratory work gives them low possibilities for development; at the same time, the results showed that possibilities for development decreases the adrenaline (beta $=-0.37$ ), noradrenaline (beta $=-2.61$ ) and dopamine (beta= -1.46) levels in the urine. Moreover, it is important to reduce exposure of workers to quantitative demands by managing their tasks more efficiently, and sensorial demands perhaps through stress management interventions.

Contrary to what is previously known from previous studies, the results of this study showed no significant associations between emotional demands and stress outcomes. The results also indicated that laboratory technicians did not experience high exposures to emotional demands and demands for hiding emotions ( $0 \%$ risk exposure), which is probably due to the fact that laboratory work is routine and involves little contact with clients. On the other hand, the results indicated that laboratory technicians consider that their work has no meaning and gives them low possibilities for development. It is also worth mentioning that very high levels of exposures to insecurity at work were reported among workers; however, the external validity of this finding requires further discussion and could not be generalized, as it is related to particularities of the country. In addition, insecurity at work 
had statistically insignificant associations with catecholamine levels in the urine.

Another curious finding of this study was the suggestion for the first time of a positive association between sensorial demands (measured by the CoPSoQ questionnaire) and adverse health events (elevated urinary catecholamine levels) among laboratory technicians. As the tasks of laboratory technicians involve maintaining alertness all of the time during daily work, studying these kinds of demands is an actual issue in laboratories. It was also noted that these demands were significantly associated with high levels of urinary catecholamine.

The findings of this study suggest that the most effective approach for reducing stress would be to advocate for more autonomy at work and to reduce sensorial demands by handling the very real problem of establishing stable relations between workers and superiors. Similarly, a key issue in improving stress in laboratory technicians is improvement of low possibilities for development and influence at work.

The present study highlights the necessity to develop practical measures by improving the occupational safety of this occupational group of workers and reducing their exposure to psychological demands.

This study has certain limitations. The sampling procedure adopted by this study (multistage random sampling) aimed at reaching a representative sample of the study population. Although the sample represented around $6 \%$ of the laboratory technicians in Lebanon, the distribution of the workers in the sample and the population were similar in terms of sex and age. Furthermore, the scores of the independent variables (work psychosocial risks) were obtained according to the participants' self-perceptions, raising another possible restriction relating to the subjectivity of the results. Another limitation could be attributed to the fact that the authors did not control for other independent variables such as intake of medicaments or the existence of a tumor (such as pheochromocytoma in adults ${ }^{21)}$ ) that could influence the urinary catecholamine values and thus possibly confound the association between the work-related psychosocial hazards and the urinary catecholamine levels.

The results of this study replicated previous research in a high risk context scarcely examined previously and reconfirmed that sensorial demands, low possibilities for development and quantitative demands were negatively associated with worker health.

\section{References}

1) Stansfeld S, Candy B. Psychosocial work environment and mental health: a meta-analytic review. Scand J Work Envirn Health 2006; 31: 443-62.

2) Borg V, Kristensen T, Burr H. Work environment and changes in self-rated health: a five year followup study. Stress Medicine 2000; 16: 37-47.

3) Karasek RA. Job demands, job decision latitude, and mental strain: implications for job redesign. Admin Sci Q 1979; 24: 285-308.

4) Johannes S. Adverse health effects of high-effort/ low-reward conditions. J Occup Health Psychol 1996; 1: 27-41.

5) Johnson S, Cooper C, Cartwright S, Donald I, Taylor P, Millet C. The experience of work related stress across occupations. J Manag Psychol 2005; 20: $178-87$.

6) Kozajda A, Szadkowska-Stańczyk I. Protection of medical diagnostic laboratory workers against biohazards. Med Pr 2011; 62: 291-5.

7) Aust B, Rugulies R, Skakon J, Scherzer T, Jensen C. Psychosocial work environment of hospital workers: validation of a comprehensive assessment scale. Int J Nurs Stud 2007; 44: 814-25.

8) Harrington JM, Shannon HS. Mortality study of pathologists and medical laboratory technicians. $\mathrm{Br}$ Med J 1975; 5992: 329-32.

9) Aziah BD, Rusli BN, Winn T, Naing L, Tengku MA. Prevalence and associated factors of job-related depression in laboratory technicians in Hospital Universiti Sains Malaysia (HUSM) and Ministry of Health Malaysia (MOH) Hospitals in Kelantan. Med J (Malaysia) 2004; 59: 268-78.

10) Medeiros WP, Setúbal S, Pinheiro PY, Dalston MO, Bazin AR, de Oliveira SA. Occupational hepatitis C seroconversions in a Brazilian hospital. Occup Med (Lond) 2012; 62: 655-7.

11) Aziah BD, Rusli BN, Winn T, Naing L, Tengku MA. Risk factors of job-related depression in laboratory technicians in Hospital Universiti Sains Malaysia (HUSM) and Kementerian Kesihatan Malaysia (KKM) hospitals in Kelantan. Southeast Asia. J Trop Med 2004; 35: 468-75.

12) Aziah BD, Rusli BN, Winn T, Naing L, Tengku MA. Prevalence and associated factors of job-related depression in laboratory technicians in Hospital Universiti Sains Malaysia (HUSM) and Ministry of Health Malaysia (MOH) Hospitals in Kelantan. Med J (Malaysia) 2004; 59: 268-78.

13) Harenstam A, Theorell T. Cortisol elevation and serum glutamyl transpeptidase in response to adverse job conditions: how are they interrelated? J Biol Psychol 1990; 31: 157-71.

14) Evolahti A, Hultcrantz M, Collins A. Women's work stress and cortisol levels: a longitudinal study of the association between the psychosocial work environment and serum cortisol. J Psychosom Res 2006; 61: 645-52.

15) Park J, Ha M, Yi Y, Kim Y. Subjective fatigue and stress hormone levels in urine according to duration of shiftwork. J Occup Health 2006; 48: 446-50.

16) Yamamoto $S$, Iwamoto $M$, Inoue $M$, Harada $N$. Evaluation of the effect of heat exposure on the autonomic nervous system by heart rate variability and urinary catecholamines. J Occup Health 2007; 
49: 199-204.

17) Kristensen TS, Hannerz H, Hogh A, Borg V. The Copenhagen Psychosocial Questionnaire (COPSOQ). A tool for the assessment and improvement of the psychosocial work environment. Scand J Work Env Health 2005; 31: 438-49.

18) Deverts DJ, Zilles K, Cohen S, Baum A. Can a 15-hour (overnight) urinary catecholamine measure substitute for a 24-hour measure? J Appl Biobehav Res 2006; 11: 69-78.

19) Boyle JG, Davidson DF, Perry CG, Connell JM. Comparison of diagnostic accuracy of urinary free metanephrines, vanillyl mandelic acid, and catecholamines and plasma catecholamines for diagnosis of pheochromocytoma. J Clin Endocrinol Metab 2007; 92: 4602-8.

20) Hansen AM, Larsen AD, Rugulies R, Garde AH, Knudsen LE. A review of the effect of the psychosocial working environment on physiological changes in blood and urine. Basic Clin Pharmacol Toxicol 2009; 105: 73-83.

21) Weinkove C. Measurement of catecholamines and their metabolites in urine. J Clin Pathol 1991; 44: 269-75. 